\title{
Neuropsychiatric Symptoms and Executive Functioning in Patients with Mild Cognitive Impairment: Relationship to Caregiver Burden
}

\author{
Kelly A. Ryan ${ }^{a} \quad$ Anne Weldon ${ }^{a} \quad$ Carol Persad $^{a} \quad$ Judith L. Heidebrink ${ }^{a, b}$ \\ Nancy Barbas ${ }^{a, b}$ Bruno Giordani ${ }^{a}$ \\ ${ }^{a}$ The University of Michigan Health System and ${ }^{b}$ Ann Arbor Veterans Affairs Medical Center, Ann Arbor, Mich., USA
}

\section{Key Words}

Mild cognitive impairment - Caregiver burden •

Neuropsychology $\cdot$ Neuropsychiatric symptoms

\begin{abstract}
Background: Caregivers of patients with mild cognitive impairment $(\mathrm{MCl})$ need similar levels of support services as Alzheimer's disease (AD) caregivers, but it is unclear if this translates to increased caregiver burden. Methods: 135 participants and their caregivers $(40 \mathrm{MCl}, 55 \mathrm{AD}$ and 40 normal controls, NC) completed questionnaires, and the patients were administered neuropsychological tests. Results: The $\mathrm{MCl}$ caregivers reported significantly more overall caregiving burden than the NC, but less than the AD. They showed similar levels of emotional, physical and social burden as the $\mathrm{AD}$ caregivers. Among the $\mathrm{MCl}$ caregivers, the neuropsychiatric symptoms and executive functioning of the patients were related to a greater burden, and the caregivers with a greater burden reported lower life satisfaction and social support, and a greater need for support services. Conclu-
\end{abstract}

\section{KARGER}

Fax +4161306 1234

E-Mail karger@karger.ch

www.karger.com (c) 2012 S. Karger AG, Basel

$1420-8008 / 12 / 0344-0206 \$ 38.00 / 0$

Accessible online at: www.karger.com/dem sion: These results indicate that $\mathrm{MCl}$ caregivers are at increased risk for caregiver stress, and they require enhanced assistance and/or education in caring for their loved ones.

Copyright $\odot 2012$ S. Karger AG, Basel

\section{Introduction}

There is extensive literature on caregiver burden by family members of patients with Alzheimer's disease (AD) [1-10], especially the physical, emotional and financial costs incurred as a result of increased caregiving responsibilities. It can be assumed that the level of caregiving burden among family members of patients with mild cognitive impairment (MCI) would be much less than that experienced by $\mathrm{AD}$ caregivers, but little research has examined this difference and little is known whether the pattern of burden among caregivers of patients with MCI is similar to that among caregivers of patients with $\mathrm{AD}$. The term MCI has been used to describe the transitional phase between normal functioning and $\mathrm{AD}[11,12]$. 
Many studies indicate that $\mathrm{MCI}$ progresses to $\mathrm{AD}$ at a relatively high rate, from 13 to $48 \%$ over $12-60$ months [3-5]. The cognitive and functional changes associated with the progression to $\mathrm{AD}$ as well as the potential for a longer disease course among individuals with MCI pose a unique set of challenges for caregivers and other family members who provide assistance to or care for these patients.

There is some newer evidence to suggest that the spouses of patients with MCI are experiencing increased caregiver burden $[13,14]$. MCI spouses/caregivers endorsed an increase in caregiving responsibilities such as greater frequency of errands, more coordination of transportation and greater management of business affairs and medications, with lifestyle constraints and an increase in household responsibilities being related to subjective caregiver burden anxiety [14]. However, no AD or other comparison groups were included and specific important patient-related characteristics such as neuropsychiatric symptoms or neuropsychological status were not examined.

Despite an abundance of literature investigating the relationship between patient cognitive functioning and burden among caregivers of $\mathrm{AD}$, few studies have examined the influence of cognitive changes found in MCI and caregiver burden. Bruce et al. [13] reported that more than $30 \%$ of the spousal or child caregivers of patients with MCI endorsed a clinically significant caregiver burden, and the level of caregiver burden was significantly associated with several patient-related factors such as increased behavioral problems, depression, memory impairments and longer course of symptoms, though the burden was not related to overall neuropsychological status. Although there was no comparison group, the pattern of findings is similar to that found in the dementia literature, but the level of burden among the MCI caregivers appeared to be lower than that found in the dementia caregivers.

Among the many symptoms associated with dementia, the presence of neuropsychiatric symptoms such as apathy, disinhibition, depression, psychosis and agitation has been found to be one of the significant predictors of caregiver burden among caregivers of patients with $\mathrm{AD}$ [15-19]. Neuropsychiatric symptoms are more common among patients with MCI compared to healthy populations [20-24], although at a lower rate than in patients diagnosed with dementia $[10,13]$. Patients with MCI who have neuropsychiatric difficulties have been found to have significantly greater cognitive and functional impairments than MCI patients without these symptoms, suggesting that the presence of neuropsychiatric symptoms is a marker for MCI severity [21], and neuropsychiatric dysfunction may exert its same effect on the family system as it does among AD patients. Despite these symptoms being commonly found in the MCI population, there have been few investigations of the influence of the neuropsychiatric symptoms of MCI patients on caregiver or spousal distress. In our prior work [25], we showed that the caregivers of individuals with MCI were already experiencing a need for increased social support services comparable to that of individuals caring for $\mathrm{AD}$ patients. Although the pattern of patient-related factors was different between the two patient groups, the level of service need for caregivers of the MCI patients was more related to the neuropsychiatric symptoms of the patients whereas the service need for $\mathrm{AD}$ caregivers was related to the patients' functional disability. Fisher et al. [26] found that although the caregivers of patients with cognitive impairment, not dementia (CIND) spent fewer hours providing active help to care for recipients than caregivers of patients with dementia, the CIND caregivers experience significant emotional strain in relation to the patients' behavioral problems and their difficulties performing instrumental activities of daily living (ADL). This pattern was slightly different than the dementia caregivers, whereby only behavioral problems predicated emotional strain and depression, and suggests a differential pattern of patient influences between the two types of caregivers. The caregivers of CIND patients also reported depressive symptoms, though at a significantly lower level than the dementia caregivers. Other research has shown that problems with ADL in patients with mild dementia, assessed using an objective performance-based measure and informant-based ratings, predict caregiver burden and caregiver psychological distress [27]. Specifically, problems with orientation, finances, transportation and communication were associated with time and developmental burden and created greater hostility in the caregivers.

It is unknown to what extent MCI family members or caregivers experience increased caregiver burden compared to $\mathrm{AD}$, despite some research suggesting that they are at an increased risk for distress and experience an increased need for social support services. Gallagher et al. [28] observed that the burden among MCI caregivers ( $\mathrm{n}=$ 17) was more likely to be predicted by patient dependence for everyday activities (both basic and independent ADL) than by patient neuropsychiatric symptoms when compared to a group of moderate-to-severely impaired patients $(n=83)$, suggesting that the onset of patient depen- 
dence in the early stages of illness is a more prevalent factor in the burden for caregivers. The identification of which factors relate to caregiver burden should provide an opportunity to intervene and possibly reduce the level of burden at the very earliest stages of a possible dementing condition, as well as demonstrate significant implications of the long-term economic challenges of the disorder as it progresses. The purpose of this study was to compare the level of caregiver burden using a multidimensional measure of caregiving burden between caregivers of patients with $\mathrm{MCI}$ and $\mathrm{AD}$ and to determine which patient or caregiver factors are most related to burden or distress. Given that neuropsychiatric symptoms are common in patients with MCI, and these same symptoms have been shown to be a predictor of caregiver burden in $\mathrm{AD}$, it was expected that such symptoms would also be related to caregiver burden among MCI caregivers. Early changes in the cognitive functioning of the patients were also predicted to be related to the level of caregiver burden.

\section{Methods}

\section{Participants}

135 participants and their study partners enrolled in the longitudinal cohort (University of Michigan - Memory and Aging Project, UM-MAP) of the University of Michigan Alzheimer's Disease Research Center (MADRC) were recruited for this study. The participants were recruited from the Cognitive Disorders Clinic in the Department of Neurology, the Neuropsychology Section at the University of Michigan, or from the community via newspaper advertisements, community outreach programs, the MADRC website, or word of mouth. Once they were screened, they were enrolled in the MADRC as part of the UM-MAP, which also includes the designation of a study partner. The participants were evaluated by a neurologist and underwent neuropsychological testing with a trained technician. Study partners completed measures of functional ability and neuropsychiatric symptoms with a trained staff member (e.g. study coordinator) and they completed a set of questionnaires containing the other measures on their own. The data of both the participants and the study partners were collected during the same research visit and all the participants provided informed consent. The UM-MAP was approved by the Institutional Human Use Review Board of the University of Michigan Health System.

Of the 135 participants (mean age $=72.4, \mathrm{SD}=9.8$ ), 40 were healthy volunteers (normal controls, NC), 40 were diagnosed with MCI and 55 with probable AD. Diagnosis of the participants, including the $\mathrm{NC}$, was done at a consensus meeting consisting of at least one neuropsychologist and two neurologists as well as other support staff. The NC were in good health and demonstrated a normal neurological examination, had no history of central nervous system disease, and had no memory-related complaints or cognitive impairments on neuropsychological testing. Partici- pants were diagnosed with MCI according to revised criteria published by Petersen [12], and participants were diagnosed with probable AD according to the NINCDS-ADRDA criteria [29]; both criteria are used in the National Alzheimer's Coordinating Center Unified Data Set funded by the National Institute on Aging [30].

Study partners were identified as a spouse, family member or close friend who knew the participant well and could rate the participant's functioning. For those participants who were designated as MCI or AD, the study partners were also identified as those individuals who provided care for the participant. Due to the inclusion of healthy control participants with no cognitive impairment or those participants with minimal cognitive impairment, not all participants were in need of a caregiver, and the term 'caregiver' is used to describe the study partners throughout this project. The caregivers were 90 women and 50 men, ranging in age from 29 to 92 years $($ mean $=65.1, \mathrm{SD}=11.7)$. The majority $(85.2 \%)$ of the caregivers identified themselves as white, 14 (10.4\%) as African American, and 2 (1.5\%) as Asian. The relationship between the caregivers and the participants included 93 (69\%) spouses, 25 (18.5\%) children, 5 (3.7\%) siblings and $6(4.5 \%)$ friends. The majority of the participant pairs (74\%) were living together at the time of the study. Most (76\%) of the caregivers reported that they had daily contact with the participant, $4.4 \%$ indicated that they had contact 3-4 days per week, $5.9 \%$ had contact 2 days per week, and $5.9 \%$ had contact fewer than 2 days per week.

\section{Measures}

Caregiver Measures

Caregiver Burden Inventory. The Caregiver Burden Inventory (CBI) [31] is a 24-item scale designed to measure the overall impact of burden on caregivers. Family members rate their feelings on a 4 -point scale $(0=$ never, $4=$ nearly always $)$ across five factors that include time dependency, development, physical health, social relationships and emotional health. Examples of items include: 'I have to help him/her with many basic functions' and 'My caregiving efforts aren't appreciated by others in my family.' Scores range from 0 to 96 points with higher scores indicating a higher caregiver burden.

Service Utilization/Service Need. The caregivers were asked to rate what kind of services they used in 'caring for the person they were with today' [32]. For each of the 18 services, there were three possible responses: $0=$ 'no, the service is not needed,' $1=$ 'yes, it is used,' and 2 = 'not used, but the service is needed.' In order to create a 'service need' variable which reflects the need for service, responses to the questions were dichotomized into the following: $0=$ service was not needed and $1=$ if the service was needed regardless if it was fulfilled or not. Scores were summed to create a total service need variable.

Satisfaction with Life Scale. The Satisfaction with Life Scale (SWLS) [33] is a five-item measure that assesses the global judgment of life satisfaction of the family member. SWLS items are global rather than specific in nature, which allows respondents to weight domains in terms of those they individually deem most important. Statements are rated on a 7-point Likert scale $(1=$ strongly disagree, $7=$ strongly agree). Examples of items on the SWLS include: 'In most ways my life is close to my ideal' and 'If I could live my life over, I would change almost nothing.'

Brief Symptom Inventory-18. The Brief Symptom Inventory-18 (BSI-18) is an 18-item, 5-point, Likert-type $(0=$ not at all, $4=$ 
extremely) scale that measures the presence of psychological symptoms (i.e. depression, anxiety and somatization) in both psychiatric and nonpsychiatric populations. The BSI-18 is a shortened version of the BSI [34], which was based on the Symptom Checklist-90-Revised [35]. Global BSI-18 scores have been reported to correlate highly ( 0.90 or greater) with analogous scores from the Symptom Checklist-90-Revised [36].

Short Form Health Survey. The Short Form 12-item Health Survey [37] is a 12-item scale that measures the overall health status of the family member. Family members are asked to rate their health, abilities to perform tasks (e.g. climbing stairs, household chores), as well as the interference of health problems in limiting their work and regular activities.

Perceived Social Support. The Social Provision Scale (SPS) is a 24 -item self-report measure completed by the family member that examines their own perceived social support [38]. Respondents indicate the extent to which six provisions (attachment, social integration, reassurance of worth, reliable alliance, guidance and opportunity for nurturance) are currently available to them, taking their entire support network into consideration. Responses are given on a 4 -point scale ( $1=$ not at all true, $4=$ completely true) to descriptions of the presence or absence of a specific provision. Total scores range from 24 (low perceived social support) to 96 (high perceived social support). Example items include: 'There are people I can depend on to help me if I really need it' and 'I feel a strong emotional bond with at least one other person.'

Neuropsychiatric Inventory Questionnaire. The Neuropsychiatric Inventory Questionnaire (NPI-Q) [39] assesses psychopathology commonly found in dementia patients through a semistructured interview with a caregiver. The version used comprises of 12 behavioral domains: delusions, hallucinations, agitation, dysphoria, anxiety, apathy, irritability, euphoria, disinhibition, aberrant motor behavior, night-time behavior disturbances, and appetite and eating abnormalities. The Individuals' symptoms were scored as 0 (absent) or 1 (present), and if present, a severity rating was obtained: 1 (mild), 2 (moderate) or 3 (severe). The range for the severity scale was $0-36$. The caregivers completed the NPI-Q with a trained staff member (e.g. study coordinator).

Functional Activities Questionnaire. The Functional Activities Questionnaire (FAQ) [40] is comprised of 10 items developed to assess instumental ADL over the previous 4 weeks (e.g. writing checks, shopping, preparing a meal, paying attention, etc). Responses range from 0 (normal) to 3 (dependent), with higher scores indicating more impairment (range $0-30$ ). The caregivers completed the FAQ with a trained staff member.

Participant Variables

Neuropsychological Assessment. All the participants were administered a battery of neuropsychological tests taken from the Unified Data Set test battery [30]. The test battery assessed a broad range of cognitive functions within a short time frame and included the Mini-Mental State Examination (MMSE) [41], Boston Naming test (30 odd-numbered items) [42], Animal Fluency [43], Logical Memory And Visual Reproduction from the Wechsler Memory Scale - Revised [44], Trail Making test (TMT) [45], Digit Symbol from the Wechsler Adult Intelligence Scale [46], the Controlled Oral Word Association test [47], the Wisconsin Card Sort test [48] and the Geriatric Depression Scale [49]. A study team neurologist completed the Clinical Dementia Rating Scale [50], which is a measure of dementia severity.

Mild Cognitive Impairment and Caregiver Burden

\section{Statistical Analyses}

We examined the summary and average scores for caregiver ratings and participant assessment variables for the different groups (NC, MCI and AD). One-way analyses of variance (ANOVAs) were employed to examine age and education differences between the groups and $\chi^{2}$ significance tests were used to examine between-group gender differences. Multivariate analysis of variance (MANOVA) was completed to examine the mean differences in caregiver burden scores between the groups. Post hoc comparisons were completed using the Scheffe F test. Product-moment correlation coefficients (Pearson's r) were computed to determine the association among sociodemographic variables, caregiver burden and other predictor variables.

All variables were screened for violations of assumptions associated with univariate and multivariate tests. Variables with nonnormal distributions that may inflate alpha were transformed to improve normality and linearity [51]. The results of this evaluation led to the square root transformation of the CBI total score, CBI subscales, the service need variable and the BSI. The untransformed values are included in the tables for ease of interpretation; however, the transformed variables are used in the statistical analyses and are noted where applicable.

\section{Results}

\section{Demographic Analyses}

There were no significant differences between the groups (NC, MCI and AD) with respect to age or educational level for either the patient participants or the caregivers (table 1), although there were significantly more female NC patients compared to AD. As might be expected based upon the selection criteria for the three groups, there was a significant difference between the patient groups on the Clinical Dementia Rating Scale, the MMSE and most of the neuropsychological variables with the exception of the Boston Naming test (see table 1). The AD group was found to have significantly greater impairments on all neuropsychological measures compared to the other two groups, with the MCI participants falling in between the AD and NC participants, although the MCI group performed no differently from the NC on the TMT Part A. The patient groups did not significantly differ from one another on the Geriatric Depression Scale. Table 1 lists the demographic information and the means and standard deviations (SD) for each group (NC, MCI and $\mathrm{AD}$ ) as well as the results of the $\chi^{2}$ tests.

\section{Multivariate Analyses}

MANOVA revealed significant differences between the NC, MCI and AD groups for the total CBI score as well as the CBI time dependency and development subscales $(\mathrm{p}<0.000)$ (see table 2$)$. Post hoc analyses showed 
Table 1. Descriptive statistics and ANOVAs for participant and caregiver demographic variables

\begin{tabular}{|c|c|c|c|c|c|c|}
\hline & $\mathrm{NC}(\mathrm{n}=40)$ & $\operatorname{MCI}(\mathrm{n}=40)$ & $\mathrm{AD}(\mathrm{n}=55)$ & $\mathrm{F}$ & $\mathrm{p}$ & Post hoc difference \\
\hline \multicolumn{7}{|l|}{ Patient } \\
\hline Age, years & $69.7(8.4)$ & $73.2(9.5)$ & $73.8(10.7)$ & 2.27 & 0.108 & \\
\hline Gender (female), $\%^{1}$ & 65.0 & 45.0 & 40.0 & 3.14 & 0.046 & $\mathrm{NC}>\mathrm{AD}$ \\
\hline \multicolumn{7}{|l|}{ Caregiver } \\
\hline Age, years & $64.7(11.4)$ & $67.3(11.1)$ & $63.7(12.2)$ & 1.13 & 0.326 & \\
\hline
\end{tabular}

Table 2. Means, SD (in parentheses) and ANOVA results for participant and caregiver variables

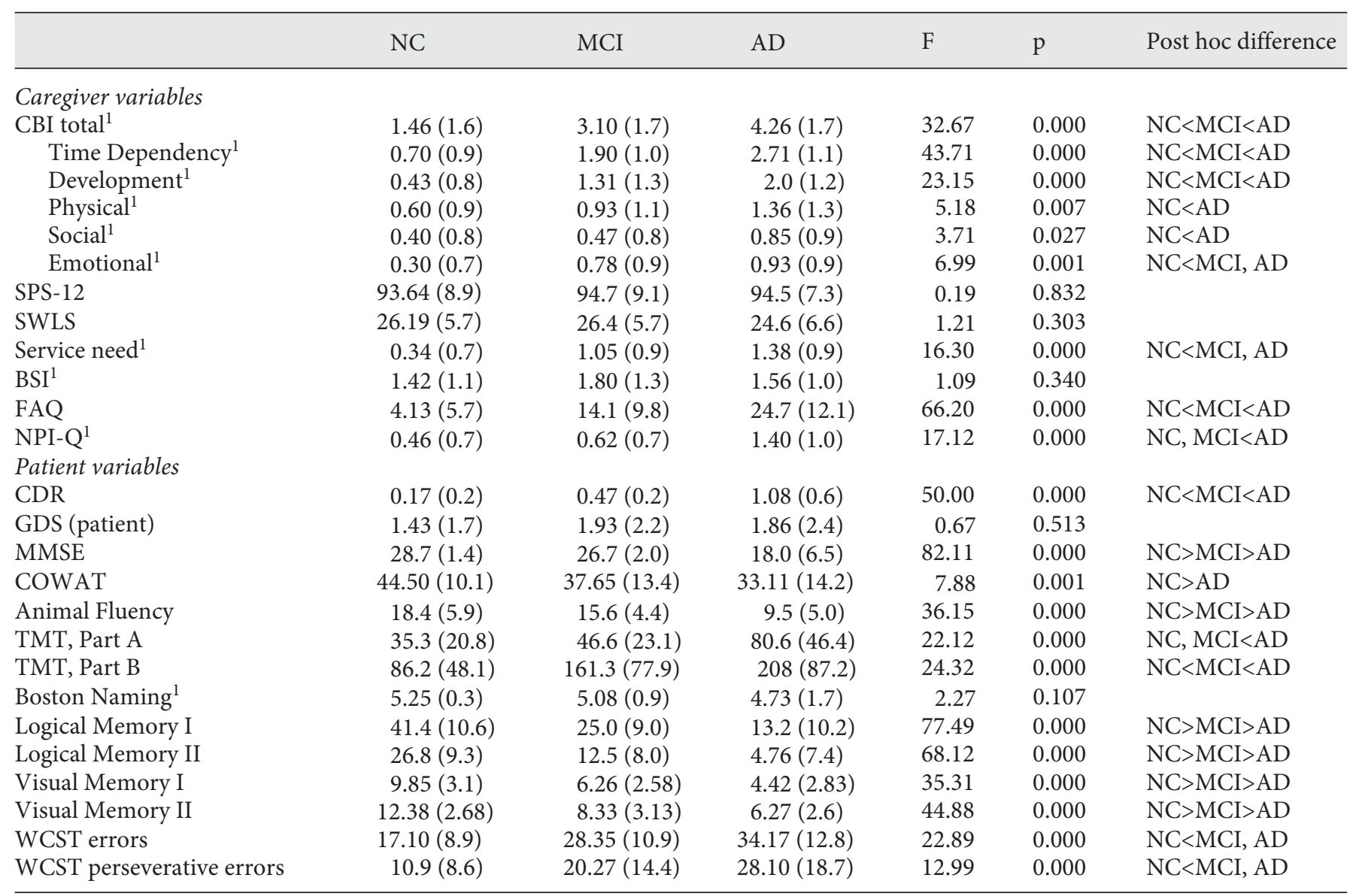

CDR = Clinical Dementia Rating; GDS = Geriatric Depression Scale; COWAT $=$ Controlled Oral Word Association test WCST = Wisconsin Card Sort test.

${ }^{1}$ Transformed variables. 


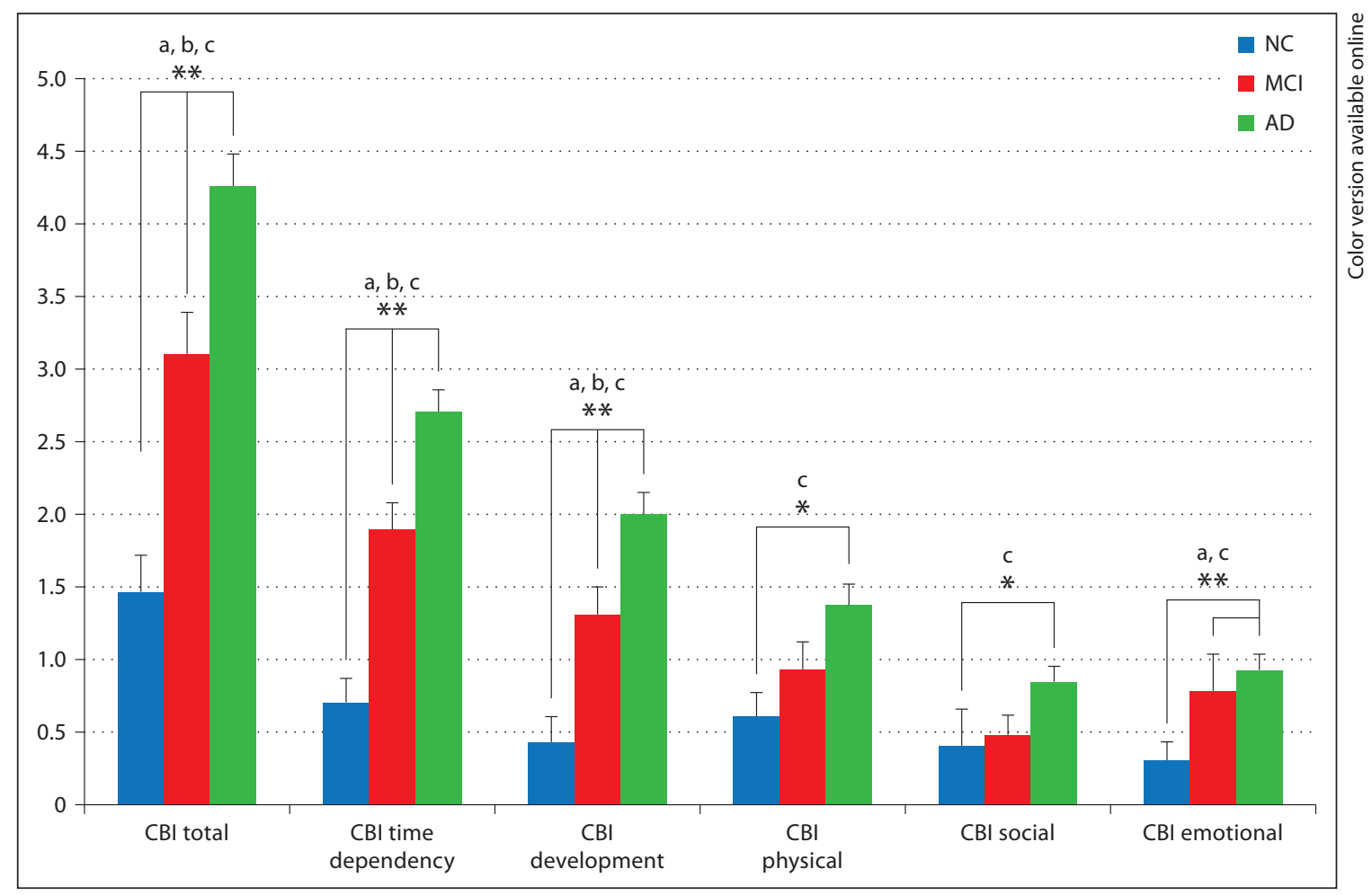

Fig. 1. Mean Scores of CBI total and subscales between NC, MCI and AD caregivers. a = Posthoc significance between NC and MCI; b = Posthoc significance between MCI and AD; $c=$ Posthoc significance between NC and $\mathrm{AD} .{ }^{*}=\mathrm{p}<0.000 ;{ }^{* *}=\mathrm{p}<0.05$.

that the $\mathrm{AD}$ group reported a significantly higher total $\mathrm{CBI}$ score and individual CBI subscale scores compared to the NC. The MCI group reported scores in between both the NC and AD groups. The MCI caregivers reported significantly more total burden than the NC, particularly in the amount of time spent caring and development subscales $(\mathrm{p}<0.000)$ from the CBI (table 2$)$. The MCI and $\mathrm{AD}$ caregivers reported similar levels of emotional strain, physical burden and social burden. Additionally, the MCI and $\mathrm{AD}$ groups reported similar levels of service need, but both caregiver groups reported significantly more service need than the NC $(\mathrm{p}<0.000)$. Figure 1 shows differences in CBI total and subscale scores between the caregiving groups and table 2 lists the means, SD and results of the MANOVA.

As expected, the $\mathrm{AD}$ patients were found to have significantly greater functional impairment (FAQ) and more neuropsychiatric symptoms (NPI-Q) then the NC and MCI patients $(\mathrm{p}<0.000)$. There were no significant caregiver group differences between caregiver reports of psychological distress (BSI), life satisfaction (SWLS) or social support (SPS-12).

\section{Relationship of MCI Total Caregiver Rating to Patient} and Caregiver Variables

Among the MCI caregivers, bivariate correlations showed a significant relationship between the CBI total score and the SPS-12 $(r=-0.368, p=0.032)$, SWLS $(r=$ $-0.408, p=0.017)$, service need $(r=0.549, p=0.001)$ and NPI-Q ( $r=0.573, p=0.000)$ (table 3). The CBI total score was significantly related to the patient's performance on the TMT Part B $(r=0.435, p=0.016)$. Among the AD caregivers, the CBI total score was related to the SWLS, service need, BSI, MMSE and Visual Memory. See table 3 for correlations between CBI total scores and measures of psychosocial, behavioral and neuropsychological variables.

\section{Discussion}

The present findings confirm that caregivers of individuals with MCI are already experiencing increased caregiver burden compared to healthy controls; in particular, they report feeling burdened by the amount of 
Table 3. Correlations between CBI Total Scores, patient variables and caregiver measures by caregiving group

\begin{tabular}{lccc}
\hline & $\begin{array}{l}\mathrm{NC} \\
(\mathrm{n}=40)\end{array}$ & $\begin{array}{l}\mathrm{MCI} \\
(\mathrm{n}=40)\end{array}$ & $\begin{array}{l}\mathrm{AD} \\
(\mathrm{n}=55)\end{array}$ \\
\hline Caregiver age & 0.27 & $0.46^{* *}$ & -0.12 \\
Caregiver education & 0.17 & -0.02 & -0.01 \\
SPS-12 & -0.25 & $-0.37^{*}$ & 0.08 \\
SWLS & -0.25 & $-0.41^{*}$ & $-0.42^{* *}$ \\
Service Need & $0.33^{*}$ & $0.55^{* *}$ & $0.39^{* *}$ \\
BSI & $0.33^{*}$ & 0.25 & $0.35^{*}$ \\
FAQ & 0.32 & 0.27 & $0.38^{* *}$ \\
NPI & $0.66^{* *}$ & $0.63^{* *}$ & $0.53^{* *}$ \\
GDS & 0.01 & 0.19 & -0.01 \\
MMSE & -0.18 & -0.04 & $-0.42^{* *}$ \\
COWAT & -0.02 & -0.21 & -0.11 \\
Animal Fluency & 0.07 & -0.13 & -0.13 \\
TMT Part A & -0.13 & 0.30 & 0.21 \\
TMT Part B & -0.02 & $0.44^{*}$ & 0.12 \\
Boston Naming & -0.10 & 0.29 & -0.08 \\
Logical Memory I & 0.03 & -0.30 & -0.22 \\
Logical Memory II & -0.01 & -0.09 & -0.19 \\
Visual Memory I & -0.11 & -0.04 & $-0.52^{* *}$ \\
Visual Memory II & $-0.34^{*}$ & 0.01 & $-0.40^{* *}$ \\
WCST errors & 0.10 & 0.15 & 0.27 \\
WCST perseverative errors & -0.03 & 0.07 & 0.20 \\
\hline & & & \\
\hline
\end{tabular}

CDR = Clinical Dementia Rating Scale; GDS = Geriatric Depression Scale; COWAT $=$ Controlled Oral Word Association test, age-adjusted percentile; WCST $=$ Wisconsin Card Sort test. ${ }^{*} \mathrm{p}<$ $0.05,{ }^{* *} \mathrm{p}<0.000$.

time spent providing care and feeling unprepared to deal with new caregiving duties in life. More specifically, the caregiving burden development subscale is likened to the perceived feelings of the caregivers about being prepared to handle a new caregiving role and feeling connected to their peers in terms of what stage of personal growth they are entering.

The results suggest that MCI caregivers are reporting that their lives have diverged from their intended course at an early stage of disease in their loved one, and that this is a prominent factor in their total perceived burden. The overall level of caregiver burden in the MCI group is greater than what the study partners of the NC are reporting, yet at a lower level than that reported by caregivers of patients with $\mathrm{AD}$, even though the MCI caregivers are reporting similar levels of emotional, physical and social burden to those caregiving for $\mathrm{AD}$ patients. Despite the diagnostic criterion for MCI being subjective cognitive complaints as well as evidence of objective cognitive impairment in the context of intact ADL and general intellectual abilities [11], there are notable symptoms in MCI that are distressing to caregivers, which are not present in the healthy controls. This provides further evidence that MCI is not always 'normal aging' and may reflect the transitional phase between normal functioning and $\mathrm{AD}$ [11]. Furthermore, the differences in caregiver burden between the diagnostic groups suggests that the level of cognitive and functional impairment often found in dementia may not be the sole factor predicting caregiving burden, as caregivers of patients with MCI who are neither cognitively or functionally impaired are experiencing notable burden. Perhaps as a consequence of increased caregiving burden, MCI caregivers are reporting a similar need for support services as AD caregivers. Even when MCI patients are in the early stages of a possible degenerative condition, their caregivers or family members are starting to experience strain in their relationships with friends/family, changes in health status, and negative feelings toward their care recipient, and at a similar level to those caring for someone with AD. These findings are particularly striking given that, by definition, the MCI patients have less severe cognitive impairments and significantly less functional impairments than the AD patients [11]. Prior research indicates that caregivers who require extra assistance when caring for patients experience significant distress or burden [52-56]. This appears to be the case with MCI caregivers, even though there is no relationship between the level of psychological distress and caregiving burden. It may be that those in the early caregiving stages of patients with MCI are recognizing the need for assistance and feeling impacted by the increased responsibility of taking care of the patient, but this has not yet interfered with psychological processes. This further indicates that early assistance and/or education in caring for their family member may provide early intervention to reduce distress associated with dementia caregiving.

Despite no difference between the emotional, physical or social burden as well as a need for support services among the MCI and AD caregivers, the factors underlying caregiver burden between the groups of caregivers are different. The neuropsychiatric symptoms of the patients and their performance on a cognitive set-shifting/executive functioning task are related to higher burden levels among the MCI caregivers. This may indicate that early neuropsychiatric or executive dysfunction among patients is an early indicator of the level of resources or intervention caregivers may require. In contrast, increased frailty, poorer visual memory and worse mental status in 
addition to neuropsychiatric symptoms are related to increased burden among $\mathrm{AD}$ caregivers. In the present study, neuropsychiatric symptoms are prevalent in participants with MCI, consistent with previous studies [20, 23]. Although the severity is lower than that reported in the $\mathrm{AD}$ participants, the neuropsychiatric symptoms and executive functioning of the MCI patients show an important relationship to the overall total burden among the MCI caregivers.

This study provides a new perspective on evaluating patients with MCI, as the findings suggest that their family members or caregivers are experiencing increased levels of burden, especially as the patient's level of neuropsychiatric or executive dysfunction increases. Future research is necessary to demonstrate that providing MCI caregivers with realistic education and available resources related to psychiatric and behavioral difficulties may significantly reduce the caregiver burden early in the course of a neurodegenerative condition. Providing caregivers or family members with access to support groups and lists of potential resources might help dispel the reportedly low levels of social support among MCI caregivers in particular, which may in turn increase their wellbeing and assist in buffering against other stresses related to caregiving.

There are limitations to this study that could impact on the interpretation and generalization of the results, such as relatively small sample sizes. Although we found significant differences between the caregiving groups using our relatively small sample sizes, we may have missed important differences or found differences that are not real based on the use of multiple tests. The present findings should be considered preliminary based on the small sample size, and replication using a larger sample is needed. Our sample included primarily highly educated Caucasian participants; therefore, the ability to generalize from the present study would be enhanced by replication with a more ethnically diverse patient and caregiver population. There is a large body of literature about how caregiver/patient ethnicity, geographic region, or relationship between the caregiver/patient impacts the caregiving burden in $\mathrm{AD}$, thus future research would need to investigate such associations in MCI caregivers. Such an educated sample might have also affected the results of cognitive testing data and, therefore, limited our analyses. In addition, data were not collected on whether the participants required the use of a caregiver and for how long they may have provided such care, though we assumed that the caregivers have been caring for patients with $\mathrm{AD}$ for a longer period of time. Certainly, the duration of care and the amount of informal care provided by the caregiver would have an impact on the overall burden and would be crucial to obtain in future studies. In addition, the recruitment of participants from mostly tertiary care specialty clinics may also limit the generalizability of the findings. Patients with MCI who present to a tertiary clinic for medical consultation may have worse or different cognitive or neuropsychiatric presentations than those in the community, thus impacting caregivers differentially. It is unclear whether similar levels of burden among MCI caregivers would be found in an exclusively community-based sample. Furthermore, it has been suggested that there may be a different clinical pattern of neuropsychiatric and psychiatric symptoms between amnestic MCI and nonamnestic MCI patients [57], which might affect the caregiver burden in different ways. Although not specifically addressed in this study, this remains an important question for further investigation and the current results might imply that MCI patients with greater executive dysfunction would be related to higher levels of caregiving burden. Future studies should examine more closely the role of cognitive performance as it relates to neuropsychiatric dysfunction in predicting caregiver burden. This is the first study to our knowledge which reports that caregivers of individuals with MCI are already experiencing increased levels of burden and that it is related to patient neuropsychiatric and executive functioning.

\section{Acknowledgements}

This research was supported by the NIH-NIA P50 AG08671 and the Michigan Alzheimer's Disease Research Center. We would like to acknowledge the National Institute on Aging Claude Pepper Older Adults Independence Center Grant AG08808.

References

$>_{1}$ Larrieu S, Letenneur L, Ogogozo JM, Fabrigoule $\mathrm{C}$, Amieva $\mathrm{H}$, Le Carret N, Bargerger-Gateau P, Dartigues JF: Incidence and outcome of mild cognitive impairment in a population-based prospective cohort. Neurology 2002;59:1594-1599.

2 Prencipe M, Santini M, Casini AR, Pezzella FR, Scaldaferri N, Culasso F: Prevalence of non-dementing cognitive disturbances and their association with vascular risk factors in an elderly population. J Neurol 2003;250: 907-912.

- 3 Unverzagt FW, Gao S, Baiyewu O, Ogunniyi AO, Gureje O, Perkins A, Emsley CL, Dickens J, Evans R, Musick B, Hall KS, Hui SL, Hendrie HC: Prevalence of cognitive impairment: data from the Indianapolis Study of Health and Aging. Neurology 2001;57:1655-1662. 
-4 Yaffe K, Fox P, Newcomber R, Sands L, Lindquist K, Dane K, Covinsky KE: Patient and caregiver characteristics and nursing home placement in patients with dementia. JAMA 2002;24:2090-2097.

5 Schultz R, O’Brien AT, Bookwala J, et al: Psychiatric and physical morbidity effects of dementia caregiving: prevalence, correlates, and causes. Gerontologist 1995;35:771-791.

-6 Dunkin J, Anderson-Hanley C: Dementia caregiver burden: a review of the literature and guidelines for assessment and prevention. Neurology 1998;51(suppl 1):S53-S60, discussion S65-S67.

7 Waite A, Bebbington P, Skelton-Robinson $\mathrm{M}$, Orrell M: Social factors and depression in carers of people with dementia. Int J Geriatr Psychiatry 2004;19:582-587.

8 Gruffydd E, Randle J: Alzheimer's disease and the psychosocial burden for caregivers. Community Pract 2006;79:15-18.

-9 Georges J, Jansen S, Jackson J, Meyrieux A, Sadowska A, Selmes M: Alzheimer's disease in real life - the dementia carer's survey. Int J Geriatr Psychiatry 2008;23:546-551.

10 Montgomery R, Kosloski K: A longitudinal analysis of nursing home placement for dependent elders cared for by spouses vs adult children. J Gerontol 1994;49:S62-S74.

- 11 Petersen RC, Doody R, Kurz A, Mohs RC, Morris JC, Rabins PV, Ritchie K, Rossor M, Thal L, Winblad B: Current concepts in mild cognitive impairment. Arch Neurol 2001;58: 1985-1992.

12 Petersen RC: Mild cognitive impairment as a diagnostic entity. J Int Med 2004;256:183-194.

13 Bruce JM, McQuiggan M, Williams V, Westervelt $\mathrm{H}$, Tremont G: Burden among spousal and child caregivers of patients with mild cognitive impairment. Dementia 2008; 25:385-390.

- 14 Garand L, Dew MA, Eazor LR, DeKosky ST, Reynolds CF: Caregiving burden and psychiatric morbidity in spouses of persons with mild cognitive impairment. Int J Geriatr Psychiatry 2005;20:512-522.

-15 Deimling G, Bass D: Symptoms of mental impairment among elderly adults and their effects on family caregivers. J Gerontol 1986; 41:778-784.

16 Pruchno RA, Resch NL: Aberrant behaviors and Alzheimer's disease: mental health effects on spouse caregivers. J Gerontol 1989; 44:S177-S182.

-17 Mangone CA, Sanguinetti RM, Baumann PD, Gonzalez RC, Pereyra S, Bozzola FG, Gorelick PB, Sica RE: Influence of feelings of burden on the caregiver's perception of the patient's functional status. Dementia 1993;4: 287-293.

-18 Rocca P, Leotta D, Liffredo C, Mingrone C, Siguado M, Capellero B, Rocca G, Simoncini M, Pirfo E, Bogetto F: Neuropsychiatric symptoms underlying caregiver stress and insight in Alzheimer's disease. Dement Geriatr Cogn Disord 2010;30:57-63.
19 Matsumoto N, Ikeda M, Fukuhara R, Shinagawa $S$, Ishikawa $T$, Mori $T$, Toyota $Y$, Matsumoto T, Adachi $\mathrm{H}$, Hirono N, Tanabe $\mathrm{H}$ : Caregiver burden associated with behavioural and psychological symptoms of dementia in elderly people in the local community. Dement Geriatr Cogn Disord 2007;23: 219-224.

20 Hwang TJ, Masterman DL, Ortiz F, Fairbanks LA, Cummings JL: Mild cognitive impairment is associated with characteristic neuropsychiatric symptoms. Alzheimer Dis Assoc Disord 2004;18:17-21.

-21 Feldman H, Scheltens P, Scarpini E, Hermann N, Mesenbrink P, Mancione L, Tekin S, Lane R, Ferris S: Behavioral symptoms in mild cognitive impairment. Neurology 2004;62:1199-1201.

22 Forsell Y, Palmer K, Fratiglioni L: Psychiatric symptoms/syndromes in elderly persons with mild cognitive impairment. Data from a cross-sectional study. Acta Neurol Scand Suppl 2003;179:25-28.

23 Lyketsos CG, Lopez O, Jones B, Fitzpatrick AL, Breitner J, DeKosky S: Prevalence of neuropsychiatric symptoms in dementia and mild cognitive impairment. JAMA 2002;88: 1475-1483.

-24 Muangpaisan W, Intalapaporn S, Assantachai P: Neuropsychiatric symptoms in the community-based patients with mild cognitive impairment and the influence of demographic factors. Int J Geriatr Psychiatry 2008;23:699-703.

25 Ryan KA, Weldon A, Huby NM, Persad C, Bhaumik AK, Heidebrink JL, Barbas N, Staffend N, Franti L, Giordani B: Caregiver support service needs for patients with mild cognitive impairment and Alzheimer disease. Alzheimer Dis Assoc Disord 2010;24: 171-176.

26 Fisher GG, Franks MM, Plassman BL, Brown SL, Potter GG, Llewellyn D, Rogers MA, Langa KM: Caring for individuals with dementia and cognitive impairment, not dementia: findings from the aging, demographics, and memory study. J Am Geriatr Soc 2011;59:488-494.

27 Razani J, Kakos B, Orieta-Barbalace C, Wong JT, Casas R, Lu P, Alessi C, Josephson $\mathrm{K}$ : Predicting caregiver burden from daily functional abilities of patients with mild dementia. J Am Geriatr Soc 2007;55:1415-1420.

28 Gallagher D, Ni Mhaolain AN, Crosby L, Ryan D, Lacey L, Coen RF, Walsh C, Coakley D, Walsh JB, Cunningham C, Lawlor BA: Dependence and caregiver burden in Alzheimer's disease and mild cognitive impairment. Am J Alzheimers Dis Other Demen 2011;26:110-114.

29 McKhann G, Drachman D, Folstein M, Katzman R, Price D, Stadlan EM: Clinical diagnosis of Alzheimer's disease: report of the NINCDS-ADRDA work group under the auspices of Department of Health and Human Services Task Force on Alzheimer's disease. Neurology 1984;34:939-944.
30 Morris J, Weintraub S, Chui HC, Cummings J, DeCarli C, Ferris S, Foster NL, Galasko D, Graff-Radford N, Peskind ER, Beekly D, Ramos EM, Kukull WA: The Uniform Data Set (UDS): clinical and cognitive variables and descriptive data from Alzheimer disease centers. Alzheimer Dis Assoc Disord 2006;20:210-216.

-31 Novak M, Guest C: Application of a multidimensional caregiver burden inventory. Gerontologist 1989;29:798-803.

32 Ho CJ, Weitzman PF, Cui XJ, Levkoff SE: Stress and service use among minority caregivers of elders with dementia. J Gerontol Soc Work 2000;33:67-88.

33 Diener E, Emmons RA, Larsen RJ, Griffin S: The Satisfaction with Life Scale. J Pers Assess 1985;49:71-75.

34 Derogatis LR, Coons HL: Self-report measures of stress; in Goldberger L, Breznitz S (eds): Handbook of Stress: Theoretical and Clinical Aspects, ed 2. New York, Free Press, 1993, pp 200-233.

35 Derogatis LR, Cleary PA: Confirmation of the dimensional structure of the SCL-90: a study in construct validation. J Clin Psychol 1977;33:981-989.

36 Derogatis LR: SCL-90-R; in Kadzin AE (ed): Encyclopedia of Psychology. Washington, American Psychological Corporation, 2000, pp 192-193.

37 Ware JE, Kosinski M, Keller SD: A 12-Item Short-Form Health Survey - construction of scales and preliminary tests of reliability and validity. Med Care 1996;34:220-233.

38 Cutrona CE, Russell D: The provisions of social relationships and adaptation stress; in Jones WH, Periman D (eds): Advances in Personal Relationships. Greenwich, JAI Press, 1987, vol 1, pp 37-68.

- 39 Kaufer DI, Cummings JL, Ketchel P, Smith V, MacMillan A, Shelley T, Lopez OL, DeKosky ST: Validation of the NPI-Q, a brief clinical form of the Neuropsychiatric Inventory. J Neuropsychiatry Clin Neurosci 2000;12: 233-239.

40 Pfeffer RI, Kurosaki TT, Harrah CH, Chance JM, Filos S: Measurement of the functional activities in older adults in the community. J Gerontol 1982;37:323-329.

41 Folstein MF, Folstein SE, McHugh PR: MiniMental State. A practical method for grading the cognitive state of patients for the clinician. J Psychiatr Res 1975;12:189-198.

42 Goodglass H, Kaplan E: The assessment of aphasia and related disorders. Philadelphia, Lee \& Ferbiger, 1983.

43 Morris JC, Heyman A, Mohs RC, Hughes JP, Vanbelle G, Fillenbaum G, Mellits ED, Clark C: The Consortium to Establish a Registry for Alzheimer's Disease (CERAD). Part I. Clinical and neuropsychological assessment of Alzheimer's disease. Neurology 1989;39: 1159-1165.

44 Wechsler D, Stone CP: Manual: Wechsler Memory Scale. New York, Psychological Corporation, 1973. 
45 Armitage SG: An analysis of certain psychological tests used in the evaluation of brain injury. Psychol Monogr 1946;60:1-48.

46 Wechsler D: Wechsler Adult Intelligence Scale-III. Cleveland, Psychological Corporation, 1997.

47 Benton A, Hamsher K: Multilingual Aphasia Examination. Iowa, University of Iowa, 1976.

48 Heaton RK: A Manual for the Wisconin Card Sorting Test. Odessa, Psychological Assessment Resources, 1981.

49 Sheikh JI, Yesavage JA: Geriatric Depression Scale (GDS): recent evidence and development of a shorter version; in Brink TL (ed): Clinical Gerontology: A Guide to Assessment and Intervention. New York, Haworth Press, 1986, pp 165-173.

50 Morris JC: The Clinical Dementia Rating (CDR): current version and scoring rules. Neurology 1993;43:2412-2414.
51 Tabachnick B, Fidell L: Using Multivariate Statistics, ed 5. New Jersey, Pearson Allyn \& Bacon, 2007.

52 Dal Santo TS, Scharlach AE, Nielsen J, et al: A stress process model of family caregiver service utilization: factors associated with respite and counseling service use. J Gerontol Soc Work 2007;49:29-49.

53 Houde SC: Predictors of elders' and family caregivers' use of formal home services. Res Nurs Health 1998;21:533-543.
54 Kosloski K, Montgomery RJ, Youngbauer J: Utilization of respite services: a comparison of users, seekers, and nonseekers. J Appl Gerontol 2001;20:111-132.

55 Rickwood DJ, Braithwaite VA: Social-psychological factors affecting help-seeking for emotional problems. Soc Sci Med 1994;39: 563-572.

56 Toseland RW, McCallion P, Gerber T, Banks S: Predictors of health and human services use by persons with dementia and their family caregivers. Soc Sci Med 2002;55: 1255-1266.

57 Rozzini L, Chilovi BV, Conti M, Delrio I, Borroni B, Trabucchi M, Padovani A: Neuropsychiatric symptoms in amnestic and nonamnestic mild cognitive impairment. Dement Geriatr Cogn Disord 2008;25:32-36. 